\title{
Colonic Epithelial Angiotensin-Converting Enzyme 2 (ACE2) Expression in Blacks and Whites: Potential Implications for Pathogenesis Covid-19 Racial Disparities
}

\author{
Mart De La Cruz ${ }^{1,2}$. David P. Nunes ${ }^{1}$ - Vaishali Bhardwaj ${ }^{1,2} \cdot$ Deepika Subramanyan $^{1,2} \cdot$ Caroline Zaworski $^{1}$. \\ Priya Roy ${ }^{1} \cdot$ Hemant K. Roy ${ }^{1,2}$ (D)
}

Received: 23 October 2020 / Revised: 16 February 2021 / Accepted: 18 February 2021 / Published online: 10 March 2021

(C) W. Montague Cobb-NMA Health Institute 2021

\begin{abstract}
Background Covid-19 toll is disproportionate in Blacks although the mechanisms remain incompletely understood. From a biological perspective, several host proteins have received most attention as logical susceptibility targets. Specifically, angiotensin-converting enzyme 2 (ACE2) serves as the epithelial cell receptor and acts in concert with transmembrane protease serine 2 (TMPRSS2). Intriguingly, ACE2 can also suppress the inflammatory response and therefore may impact the severity of Covid-19 infections (from the exuberant immune response a.k.a. "cytokine storm"). We, therefore, assessed expression of ACE2 and TMPRSS2 in Blacks versus Whites.

Methods Archived mucosal biopsies from colonoscopic biopsies of visually normal rectal mucosa without concurrent neoplasia or inflammation were used for this study. Total mRNA was isolated and subjected to real-time polymerase chain reaction for ACE2, and TMPRSS2 was assessed from non-Hispanic Blacks $(n=45)$ and non-Hispanic Whites $(n=38)$. GAPDH and betaactin were used for normalization. Multivariable analysis was performed using Analyse-IT software.

Results ACE2 and TMPRSS2 levels were not altered by gender, BMI, or age. ACE2 levels were lower in Blacks than Whites achieving statistical significance in multivariable $(0.51$-fold, $p=0.03)$ but not quite in univariable $(p=0.07)$ analysis. This downregulation was mirrored in TMRPSS2 in both univariable $(p=0.03)$ and multivariable analyses $(0.41$-fold, $p=0.02)$. Moreover, there was a strong correlation between ACE2 and TMPRSS2 levels $(r$-squared $=0.78)$.

Conclusions To our knowledge, this is the first report on racial differences inACE2 and TMPRSS2 mucosal expression. This may provide potential biological underpinnings for the disproportionately higher mortality of Covid-19 in Blacks and should spur future studies.
\end{abstract}

Keywords Covid-19 - ACE2 $\cdot$ Racial disparities $\cdot$ TMPRSS2

\section{Introduction}

The Covid-19 pandemic has had a devastating impact globally with regard to both morbidity and mortality. The spectrum of infection spans the gamut of asymptomatic infection to fulminant disease (e.g., acute respiratory distress syndrome), and prediction of disease course remains challenging. In Western countries, one striking feature has been the disproportionate

Hemant K. Roy

hemant.roy@bcm.edu

1 Section of Gastroenterology, Boston University Medical Center, Boston, MA 02118, USA

2 Department of Medicine, Baylor College of Medicine, Houston, TX 77030, USA toll in racial and ethnic minorities. For instance, the incidence of Covid-19 is more than 3-fold higher in predominantly Black versus White populations in the USA [1]. Moreover, in predominantly Black counties, the death rate was 6-fold higher than noted in counties that are largely White [1]. Like other health-related racial disparities, the pathogenesis for Covid-19 is likely multifactorial with both socioeconomic and biological contributors [2]. However, the biological underpinnings in racial Covid-19 mortality differences remain largely unexplored.

The molecular mechanisms for infection with the novel Coronavirus virus requires the viral envelop Spike protein to interact the host cell surface receptor, angiotensin-converting enzyme 2 (ACE 2) [3]. From the virus perspective, there is evidence that alterations in the Spike protein may modulate infectivity [4]. On the host side, in order for the Spike protein 
to bind to ACE2, it requires modification with the host transmembrane protease serine 2 (TMPRSS2) [3]. Interestingly, ACE2 has shown to be necessary for infection, and hence, lower levels of ACE2 are associated with decreased risk of developing infection (e.g., in pediatric population) [5]. On the other hand, several lines of evidence indicate that ACE2 levels might be inversely correlated with Covid-19 infection severity. The rationale may be that most significant/fulminant infections are driven by triggering an overactive immune system ("cytokine storm"). Recent data has suggested that ACE2 might dampen the exuberant immune response that perpetuates a variety of lung injuries including adult respiratory distress syndrome (ARDS) [6, 7]. ACE2 is seen throughout the epithelium and is expressed in the GI tract [8] which may be one of the reasons for frequent gastrointestinal symptoms in Covid-19 infections [9].

We postulated the racial difference in ACE2/TMPRSS2 epithelial expression as a factor in Covid-19 racial disparities. We used a sample of convenience (previously rectal mucosal biopsies) and assessed ACE2/TMPRSS2 mRNA in healthy controls to ascertain whether Blacks and Whites had different expression.

\section{Materials and Methods}

\section{Clinical Samples}

All human studies were done under the auspices and approval of the Boston University Institutional Review Board. For this study, we utilized biopsies obtained from 84 non-Hispanic Blacks and Whites undergoing colonoscopy for colon cancer screening and surveillance at Boston Medical Center who were without neoplasia/inflammation obtained at colonoscopy. We chose neoplasia-free patients to avoid any potential confounding from field carcinogenesis. Aside from colonoscopic findings, clinical information that was available included self-reported race and gender along with body mass index (BMI). No data was available on variables such as hypertension and medications including angiotensin receptor blockers (ARBs) and angiotensin-converting enzyme (ACE) inhibitors. Exclusion criteria included incomplete colonoscopy or failure to visualize colon (poor preparation), evidence of colitis or rectal mucosal abnormalities, and coagulopathy/ immunosuppression which would preclude biopsies.

\section{RT-PCR}

Biopsies were collected in PBS and snap frozen in liquid nitrogen for RNA preservation. To isolate RNA, biopsies were homogenized using BeadBug microtube homogenizer (Millipore Sigma, St. Louis, MO) and then subjected to the trizol-based RiboPure RNA Purification Kit (Thermo Fisher
Scientific, Waltham, MA) using manufacturer's instructions. cDNA was reverse-transcribed using high-capacity cDNA synthesis kit (Thermo Fisher Scientific, Waltham, MA). RTPCR was performed using TaqMan real-time PCR assays (Thermo Fisher Scientific, Waltham, MA) for genes to ACE2 and TMPRSS2. Target genes were normalized using the average of the widely accepted internal loading controls (house-keeping genes) average of internal control genes glyceraldehyde-3-phosphate dehydrogenase (GAPDH) and beta-actin for enhanced validity and reproducibility of RTPCR analysis.

\section{Statistical Analysis}

Data are expressed at cycle threshold (CT) value normalized to the mean of GAPDH and beta-actin CT values as controls. Relative mRNA expression between Blacks and Whites was normalized to expression in Whites. Chi-square test was used to compare categorical data and continuous data compared using Student's $t$-test or Wilcoxon Mann-Whitney test. Multivariate analysis was performed using linear regression analysis. A $p$ value $<0.05$ was deemed significant. All analyses were performed using Analyse-It software (Leeds UK).

\section{Results}

The cohort was representative of our safety net hospital colon cancer screening/surveillance program. As noted in Table 1, the Black and White cohort appeared well matched with regard to gender, smoking status (ever smoker versus never smoker), diabetes, or BMI although Blacks were slightly younger consistent with the recent practice of screening Blacks at an earlier age for colon cancer screening (4.2 year, $p=0.01)$ [10].

We assessed the rectal mRNA expression ACE2 and TMPRSS2 in our cohort expression comparing clinical variables implicated in Covid-19 severity. In Table 2, we utilized cycle threshold (CT) PCR value which are inverse of mRNA

Table 1 Demographic characteristic of Black and White cohorts. Blacks appeared to be slightly younger $(p=0.01)$ but no difference in gender, body mass index (BMI), diabetes, and smoking history (ever versus never users)

\begin{tabular}{llll}
\hline & Black $(n=45)$ & White $(n=38)$ & $p$ value \\
\hline Age mean (sd) & $54.4(6.8)$ & $58.6(7.6)$ & 0.014 \\
Gender male (\%) & $20(44 \%)$ & $17(45 \%)$ & 0.979 \\
BMI (sd) & $31.5(7.8)$ & $31.1(10.2)$ & 0.867 \\
DM (\%) & $12(27 \%)$ & $6(16 \%)$ & 0.231 \\
Smoker (\%) & $12(27 \%)$ & $10(26 \%)$ & 0.971 \\
\hline
\end{tabular}


Table 2 ACE2 and TMPRSS2 cycle threshold (inverse of mRNA expression) in mucosal biopsy samples for dichotomous variables. Data expressed in median and interquartile range (IQR)

\begin{tabular}{llllll}
\hline & \multirow{2}{*}{ ACE2 } & & & \multicolumn{2}{l}{ TMPRSS2 } \\
\cline { 2 - 3 } \cline { 5 - 6 } & Median (IQR) & $p$ value & & Median (IQR) & $p$ value \\
\hline Gender & & & & \\
Male & $7.48(3.69)$ & 0.84 & & $2.87(4.29)$ & 0.74 \\
Female & $7.74(3.81)$ & & $3.69(4.59)$ & \\
Diabetes & & & & \\
No & $7.64(3.70)$ & 0.84 & $3.57(4.54)$ & 0.60 \\
Yes & $7.54(4.41)$ & & $2.19(4.82)$ & \\
Smoking & & & & \\
No & $7.60(3.81)$ & 0.99 & $3.57(4.77)$ & 0.17 \\
Yes & $7.71(3.72)$ & & $2.37(4.24)$ & \\
\hline
\end{tabular}

expression (i.e., the more PCR cycles needed to detect a signal from a gene, the less starting mRNA is present). There was no difference in either ACE2 or TMPRSS2 levels with gender, diabetes, or smoking. Body mass index (BMI) showed a weak correlation with TMPRSS2 $(r$-squared $=0.068, p=0.02)$ with no other non-race variable including age found to be significant.

We next focused on race (self-reported) and noted that the median CT values for ACE2 in Blacks was 7.88 compared to that in Whites which was 6.21, indicating lower gene expression. Figure 1 a shows mRNA expression in a box and whisker plot. The $p$-value was 0.07 approaching but not achieving conventionally statistical significance thresholds which may be due to the biological variability in ACE2 expression. Indeed, the interquartile range (IQRs) were large in both the Black and especially the White cohorts (IQRs $=2.34$ and 4.18, respectively). TMPRSS2 (Fig. 1b) mirrored ACE2 levels with a dramatic downregulation with $\mathrm{CT}$ value with Whites being $4.16(\mathrm{IQR}=4.07)$ and Blacks was $0.52(\mathrm{IQR}=4.29)$ and this achieved statistical significance $p$-value of 0.03 .

We next performed multivariable analysis of the demographic variables to further ensure that our findings of ACE2 and TMPRSS2 decrease in Blacks were not simply a result of confounding by co-segregating risk factors. After controlling for the factors obtained from patients (Table 1), we observed that both ACE2 $(p=0.03)$ and TMPRSS2 $(p=0.02)$ independently correlated with race. When the data was normalized to the Whites, mean ACE2 and TMPRSS2 expression were 0.51- and 0.41-fold, respectively, lower in Blacks (Table 3).

Finally, we evaluated the correlation between ACE2 and TMPRSS2 expression in the colonic mucosa. As noted in Fig. 2, TMPRSS2 expression was generally higher than ACE2. There was a close correlation on a per-individual basis; there was a strong correlation between the two in the colon $(r$ squared of 0.78 by Pearson).

\section{Discussion}

To our knowledge, this is the first report on ACE2 expression in Blacks versus Whites from normal epithelial cell. We noted that Blacks had lower levels of both ACE2 and TMPRSS2 expression. While the ACE2 approached but did not quite achieve conventional statistical significance, reassuringly using a multivariable model (with age, gender), it crossed the statistical significance threshold. Furthermore, ACE2 closely mirroring with TMPRSS2 which did achieve statistical significance in both the univariable and multivariable analyses.

The epidemiological data for racial disparities in Covid-19 is compelling. For instance, in a large cohort study in Louisiana, Blacks with Covid-19 had a relative risk of 1.96 (95\% confidence interval 1.62-2.37) of being hospitalized when compared to Whites after adjusting for covariates (obesity, age, sex, Charlson Comorbidity Index score, residence in a low-income area, and insurance plan) [11]. Similarly, Azar and colleagues noted that in 1052 confirmed cases, nonHispanic Blacks had a 2.7-fold higher risk of hospitalization than non-Hispanic Whites even after adjusting for age, gender, comorbidities, and income [12]. However, it is possible that there may be residual confounding that might occur from socioeconomic factors (living conditions, essential worker designation etc.). Racial health disparities (cancer, hypertension, etc.) are generally related to both biological and socioeconomic factors [2].

ACE2 is well established as the molecular receptor for the novel Coronavirus. Using transgenic mice, it has been shown that eliminating ACE2 markedly suppressed infections of Covid-19. Thus, at first pass, ACE 2 data may seem paradoxical. However, there is emerging evidence that ACE2 downregulation may potentiate the severity of inflammation which is a trigger towards ARDs and thus mortality from Covid-19. ACE 2 is responsible for degrading a variety of proinflammatory peptides such as angiotensin II. Increased angiotensin II leads to enhanced inflammation including macrophage activation with concomitant elaboration of interleukin 6, tumor necrosis factor alpha, and other inflammatory cytokines [6]. Moreover, ACE2 also generates angiotensin 1-7 which has salutary effects interacting with the Mas receptor to counteracting the pro-inflammatory effects of angiotensin II [6]. Rivellese and colleagues point out that ACE2 deficiency may occur in the elderly providing one potential mechanism for the higher mortality from Covid-19 [7]. Indeed, in experimental models of pneumonia, ACE2 was protective against severe inflammation induced by acid [13]. Furthermore, the powerful survival advantage effects of immune suppressants including the corticosteroid dexamethasone underscore the hyper-immune response [14]. As pointed out by Zamai, the role of ACE is complex with an "Adverse Axis" and the ACE2 "Protective Axis" with Covid-19 infection disrupting 
Fig. 1 Impact of race on rectal ACE2 and TMPRSS2 expression. $\mathrm{CT}$ values (inverse of gene expression) normalized to the mean of the Whites. Box and whisker plot, median, and IQR, blue line mean. a ACE2: Blacks had a trend towards higher $\mathrm{CT}$ values (inverse of gene expression) and hence lower gene expression when compared to Whites $(p=0.072)$. b TMPRSS2: Blacks had a higher $\mathrm{CT}$ values (inverse of gene expression) and hence lower gene expression when compared Whites $(p=$ 0.028 )

\section{a}
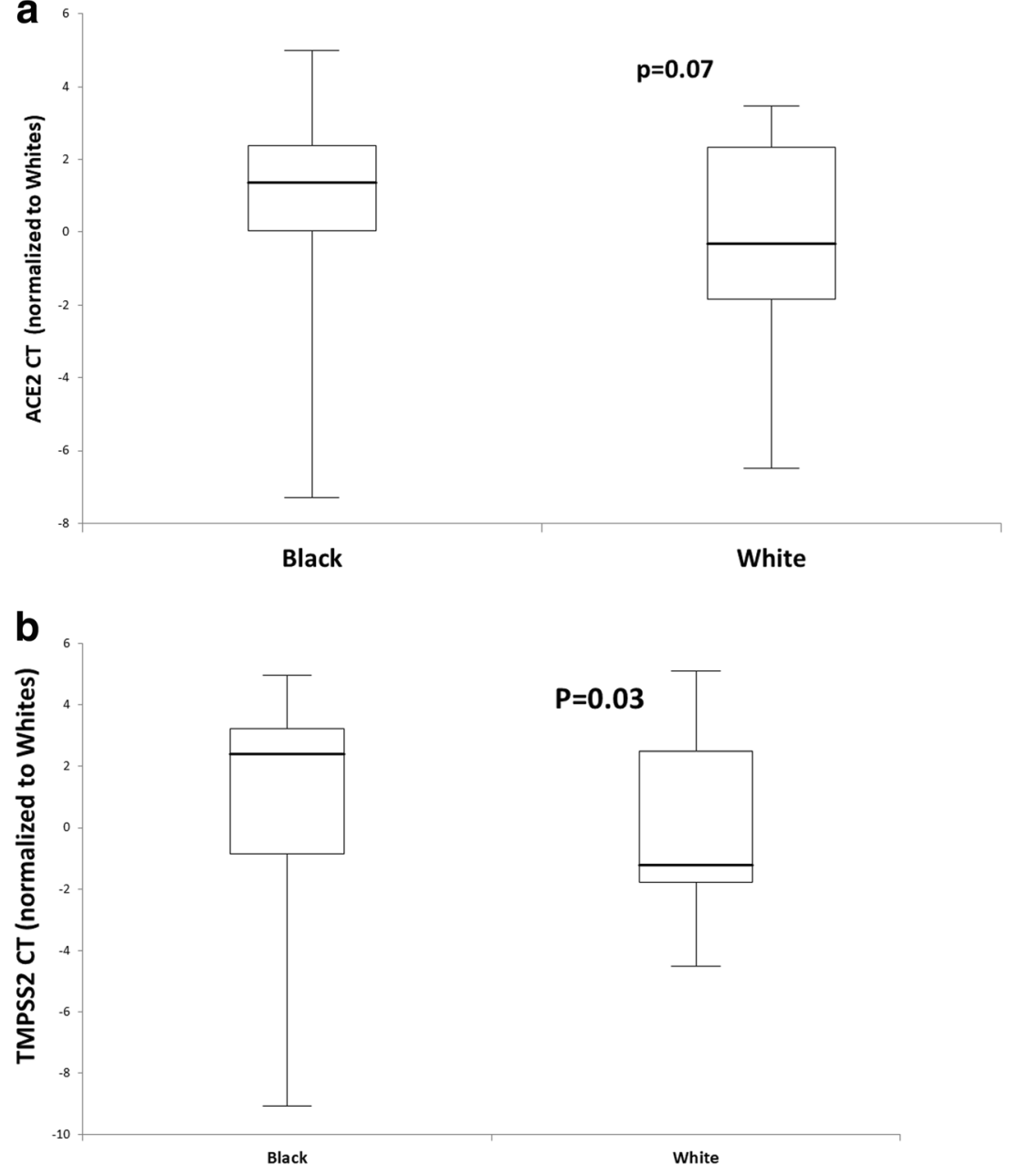

the Yin-Yang leading to the vasoconstriction, inflammation etc. that characterizes serious/lethal infections [15].

One important caveat is that the epidemiology suggests that both the case fatality rate and prevalence of infection are higher. Our data suggests that ACE2 suppression may increase severity but would not explain increased prevalence of Covid-19 in Blacks. Indeed, since ACE2 is necessary for Covid-19 access to epithelium, this downregulation in Blacks would be predicted to and loss of ACE2 seems to decrease risk of infection. Similarly, since TMPRSS2 acts on the coronavirus Spike protein to allow virus internalization and thus suppression would also be anticipated to decrease risk of

Table 3 Multivariable analysis of ACE2 and TMPRSS2 mRNA in Blacks expressed as \% of White. This was adjusted for age, BMI, diabetes, and smoking. Both ACE2 and TMPRSS2 were significantly decreased

\begin{tabular}{llc}
\hline & mRNA expression in Blacks (\% White) & $p$ value \\
\hline ACE2 & $51 \%$ & 0.03 \\
TMPRSS2 & $41 \%$ & 0.02 \\
\hline
\end{tabular}

infection. Interestingly, ACE2 rectal levels did not parallel other Covid-19 severity risk factors (obesity, age, smoking status etc.) leading to the speculation of distinct modalities involved in racial differences [16].

The mechanisms of rectal mucosal ACE2 downregulations in Blacks is unclear. The determinants of ACE2 levels may be influenced by genetics (e.g., single-nucleotide polymorphisms) and epigenetics (e.g., methylation) both of which have shown to be common themes in racially distinct gene expression. From a genetic perspective, there are a number of single-nucleotide polymorphisms (SNPs) that have been shown to racially segregate [17]. SNPs can lead to altered protein levels through alterations in regulatory units, splice sites etc. Additionally, ACE2 levels have been shown to be impacted by epigenetics including methylation [18]. For SNPs which are clearly racially mediated, several lines of evidence indicate that racially mediated methylation is noted in cancer disparity studies [19].

These findings are consistent with data from Chen et al. where aging, a key risk factor for severity, was associated with a decreased tissue ACE2 level [20]. Our data is in contradistinction to ACE2 increase in sputum of asthmatics which 
Fig. 2 Correlation between ACE2 and TMPRSS2 in per patient colonic mucosa. Patient ACE2 and TMPRSS2 cycle threshold was closely correlated (Pearson's $r=0.78$ )

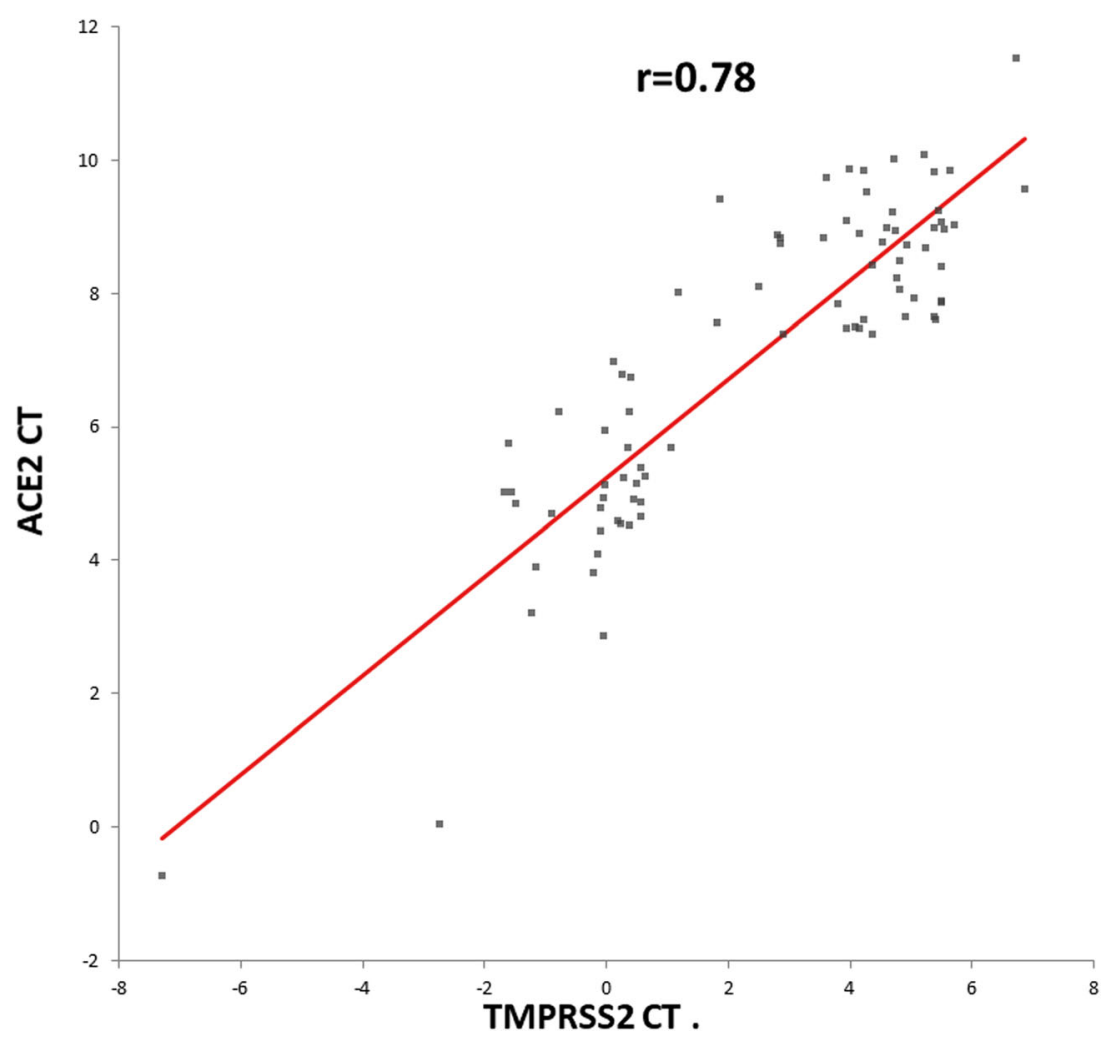

showed higher ACE2 expression [21]. However, in this study, once patients were given inhaled corticosteroids, the racial difference abated suggesting this was a result of inflammatory rather than epithelial expression but this was removed with inhaled steroids suggesting non-epithelial drivers [21].

For the use of rectal biopsies as a sample of convenience, we acknowledge that bronchial epithelium might be more relevant. However, GI manifestations are frequent $(\sim 20 \%$ in Covid-19 infections) and includes diarrhea, vomiting, and anorexia. Additionally, $\sim 50 \%$ of patients have viral RNA detectable in feces. Moreover, there is evidence of GI manifestations frequently parallel in overall disease [9]. With regard to ACE2, its expression at both the mRNA and protein level in intestinal tissue is strongly relative to many other sites [8,22]. While it is logical to extrapolate that the processes that drive ACE2 expression (SNPs, methylation) in the GI tract should have a more ubiquitous impact, this needs to be empirically corroborated. In this regard, some recent data suggests that factors that may impact ACE2 levels (age, obesity etc.) impact multiple epithelial sites [20]. Moreover, recent single cell RNA sequencing study showed co-expression of ACE2 and TMPRSS2 mirrored in several tissue types including respiratory tree and colon [23]. These authors also demonstrate that TMPRSS2 was only expressed in a subset of ACE2-positive cells supporting the role of other proteases and indicating the primacy of ACE2 for Covid-19 infection [23].

Other limitations include the modest sample size which precluded univariable $(p=0.07)$ analysis achieving statistical significance, but the multivariable analysis is reassuring. Other putative confounders such as hypertension or medications which may modulate ACE2 expression (e.g., ARBs have been shown to increase cardiac ACE2 levels) were unable to be assessed [24]. In addition, the relative importance of these biological factors versus socioeconomic remains to be determined [25]. Blacks are known to have many more underlying medical issues associated with Covid-19 susceptibility (obesity, diabetes, hypertension, etc.) [2]. While our data used multivariable analysis to address confounders, we were only able to adjust for a limited number of variables leading to the possibility of unmeasured confounders. From a socioeconomic perspective, issues like more frequency of needing to work (essential workers in pandemic) and less ability to socially distance also may play a role in this disparity. However, there is precedence for biological factors involved with Covid-19 severity. For instance, a recent genome-wide association study indicated that blood types are associated with severity risk of Covid19 [26]. Importantly, it is well established that there are significant differences in blood types by race [27]. It needs to be reiterated that our data does not indicate the relative contributions of biological versus socioeconomic factors in the higher mortality of Covid-19 in Blacks. Indeed, it is conceivable that socioeconomic and biological factors may act in combination to foster Covid-19 disparities through the chronic downregulation of ACE2 expression leading to more severe sequelae of infection. 
In summary, we demonstrate that ACE2 levels are decreased in Blacks providing the potential insights in biological underpinnings of Covid-19 racial disparities. This may offer new vistas in mitigating Covid-19 toll on vulnerable populations (i.e., potential druggable pathways) which needs to be implemented in conjunction with efforts targeting the socioeconomic and co-segregating risk factors (obesity, hypertension, smoking etc.) which are likely contributors to these disparities.

\section{Code Availability Not applicable}

Author Contribution
$\begin{array}{ll}\text { De La Cruz } & \text { data acquisition, preparation of manuscript } \\ \text { Nunes } & \text { data analysis, preparation of manuscript } \\ \text { Bhardwaj } & \text { data acquisition } \\ \text { Subramanyan } & \text { data acquisition } \\ \text { Zaworski } & \text { data acquisition } \\ \text { P. Roy } & \text { data acquisition } \\ \text { H. Roy } & \text { study idea, design, data analysis, preparation of manuscript }\end{array}$

Funding This work was supported in part by grants from the National Institutes of Health: R01CA22491, R33CA225323, R21MD013631

Data Availability The data and material are available by contacting the corresponding author.

\section{References}

1. Yancy CW. COVID-19 and African Americans. JAMA. 2020;323(19):1891-2. https://doi.org/10.1001/jama.2020.6548.

2. Physicians ACo. Racial and ethnic disparities in health care. American College of Physicians. 2010.

3. Hoffmann M, Kleine-Weber H, Schroeder S, Kruger N, Herrler T, Erichsen S, et al. SARS-CoV-2 cell entry depends on ACE2 and TMPRSS 2 and is blocked by a clinically proven protease inhibitor. Cell. 2020;181(2):271-80 e8. https://doi.org/10.1016/j.cell.2020. 02.052 .

4. Korber B, Fischer WM, Gnanakaran S, Yoon H, Theiler J, Abfalterer W, et al. Tracking changes in SARS-CoV-2 Spike: evidence that D614G increases infectivity of the COVID-19 virus. Cell. 2020;182(4):812-27 e19. https://doi.org/10.1016/j.cell.2020. 06.043 .

5. Patel AB, Verma A. Nasal ACE2 levels and COVID-19 in children. JAMA. 2020;323(23):2386-7. https://doi.org/10.1001/jama.2020. 8946.

6. Verdecchia P, Cavallini C, Spanevello A, Angeli F. The pivotal link between ACE2 deficiency and SARS-CoV-2 infection. Eur J Intern Med. 2020;76:14-20. https://doi.org/10.1016/j.ejim.2020.04.037.

7. Rivellese F, Prediletto E. ACE2 at the centre of COVID-19 from paucisymptomatic infections to severe pneumonia. Autoimmun Rev. 2020;19(6):102536. https://doi.org/10.1016/j.autrev.2020. 102536.

8. Hikmet F, Mear L, Edvinsson A, Micke P, Uhlen M, Lindskog C. The protein expression profile of ACE2 in human tissues. Mol Syst Biol. 2020;16(7):e9610. https://doi.org/10.15252/msb.20209610.

9. Cheung KS, Hung IFN, Chan PPY, Lung KC, Tso E, Liu R, et al. Gastrointestinal manifestations of SARS-CoV-2 infection and virus load in fecal samples from a Hong Kong Cohort: systematic review and meta-analysis. Gastroenterology. 2020;159(1):81-95. https:// doi.org/10.1053/j.gastro.2020.03.065.

10. Williams R, White P, Nieto J, Vieira D, Francois F, Hamilton F. Colorectal cancer in African Americans: an update. Clin Transl Gastroenterol. 2016;7(7):e185. https://doi.org/10.1038/ctg.2016. 36.

11. Price-Haywood EG, Burton J, Fort D, Seoane L. Hospitalization and mortality among Black patients and White patients with Covid19. N Engl J Med. 2020;382(26):2534 43. https://doi.org/10.1056/ NEJMsa2011686.

12. Azar KMJ, Shen Z, Romanelli RJ, Lockhart SH, Smits K, Robinson $\mathrm{S}$, et al. Disparities in outcomes among COVID-19 patients in a large health care system in California. Health Aff (Millwood). 2020;39(7):1253-62. https://doi.org/10.1377/hlthaff.2020.00598.

13. Imai Y, Kuba K, Rao S, Huan Y, Guo F, Guan B, et al. Angiotensin-converting enzyme 2 protects from severe acute lung failure. Nature. 2005;436(7047):112-6. https://doi.org/10.1038/ nature03712.

14. Group RC, Horby P, Lim WS, Emberson JR, Mafham M, Bell JL, et al. Dexamethasone in hospitalized patients with Covid-19preliminary report. N Engl J Med. 2020. https://doi.org/10.1056/ NEJMoa2021436.

15. Zamai L. The Yin and Yang of ACE/ACE2 pathways: the rationale for the use of renin-angiotensin system inhibitors in COVID-19 patients. Cells. 2020;9(7). https://doi.org/10.3390/cells9071704.

16. Mehra MR, Desai SS, Kuy S, Henry TD, Patel AN. Cardiovascular disease, drug therapy, and mortality in Covid-19. N Engl J Med. 2020;382(25):e102. https://doi.org/10.1056/NEJMoa2007621.

17. Hou Y, Zhao J, Martin W, Kallianpur A, Chung MK, Jehi L, et al. New insights into genetic susceptibility of COVID-19: an ACE2 and TMPRSS2 polymorphism analysis. BMC Med. 2020;18(1): 216. https://doi.org/10.1186/s12916-020-01673-z.

18. Fan R, Mao SQ, Gu TL, Zhong FD, Gong ML, Hao LM, et al. Preliminary analysis of the association between methylation of the ACE2 promoter and essential hypertension. Mol Med Rep. 2017;15(6):3905-11. https://doi.org/10.3892/mmr.2017.6460.

19. Wang X, Ji P, Zhang Y, LaComb JF, Tian X, Li E, et al. Aberrant DNA methylation: implications in racial health disparity. PLoS One. 2016;11(4):e0153125. https://doi.org/10.1371/journal.pone. 0153125 .

20. Chen J, Jiang Q, Xia X, Liu K, Yu Z, Tao W et al. Individual variation of the SARS-CoV-2 receptor ACE2 gene expression and regulation. Aging Cell. 2020;19(7). https://doi.org/10.1111/ acel.13168.

21. Peters MC, Sajuthi S, Deford P, Christenson S, Rios CL, Montgomery MT, et al. COVID-19-related genes in sputum cells in asthma. Relationship to Demographic Features and Corticosteroids. Am J Respir Crit Care Med. 2020;202(1):83-90. https://doi.org/10.1164/rccm.202003-08210C.

22. Hamming I, Timens W, Bulthuis ML, Lely AT, Navis G, van Goor $\mathrm{H}$. Tissue distribution of ACE2 protein, the functional receptor for SARS coronavirus. A first step in understanding SARS pathogenesis. J Pathol. 2004;203(2):631-7. https://doi.org/10.1002/path. 1570.

23. Sungnak W, Huang N, Becavin C, Berg M, Queen R, Litvinukova $\mathrm{M}$, et al. SARS-CoV-2 entry factors are highly expressed in nasal epithelial cells together with innate immune genes. Nat Med. 2020;26(5):681-7. https://doi.org/10.1038/s41591-020-0868-6.

24. Oz M, Lorke DE, Kabbani N. A comprehensive guide to the pharmacologic regulation of angiotensin converting enzyme 2 (ACE2), the SARS-CoV-2 entry receptor. Pharmacol Ther. 2020;221: 107750. https://doi.org/10.1016/j.pharmthera.2020.107750.

25. Chowkwanyun M, Reed AL Jr. Racial health disparities and Covid19-caution and context. N Engl J Med. 2020;383(3):201-3. https:// doi.org/10.1056/NEJMp2012910. 
26. Ellinghaus D, Degenhardt F, Bujanda L, Buti M, Albillos A, Invernizzi $\mathrm{P}$, et al. Genomewide association study of severe Covid-19 with respiratory failure. N Engl J Med. 2020;383:152234. https://doi.org/10.1056/NEJMoa2020283.

27. Garratty G, Glynn SA, McEntire R. Retrovirus Epidemiology Donor $\mathrm{S}$. $\mathrm{ABO}$ and $\mathrm{Rh}(\mathrm{D})$ phenotype frequencies of different racial/ethnic groups in the United States. Transfusion. 2004;44(5): 703-6. https://doi.org/10.1111/j.1537-2995.2004.03338.x.

Publisher's Note Springer Nature remains neutral with regard to jurisdictional claims in published maps and institutional affiliations. 